\title{
Dosimetric Comparison of Noncoplanar and Coplanar Volumetric Modulated Arc Therapy Plans for Esophageal Cancer
}

\author{
So-Yeon Park \\ Department of Radiation Oncology, Veterans Health Service Medical Center, Seoul, Korea
}

Received 25 November 2020 Revised 15 December 2020

Accepted 21 December 2020

Corresponding author

So-Yeon Park

(vsoyounv@gmail.com)

Tel: 82-2-2225-4648

Fax: 82-2-2225-4640
Purpose: We compared noncoplanar volumetric modulated arc therapy (ncVMAT) plans to coplanar VMAT (cVMAT) plans by evaluating the dosimetric quality of each for esophageal cancer.

Methods: Twenty patients treated for esophageal cancer with the CVMAT technique were retrospectively selected. The cVMAT plans consisted of three coplanar full arc beams. The ncVMAT plans consisted of two coplanar full arc beams and one noncoplanar partial arc beam ranging from $45^{\circ}$ to $315^{\circ}$ with a couch rotation angle of $315^{\circ} \pm 5^{\circ}$. For dosimetric evaluation, the dose-volumetric (DV) parameters of the planning target volume (PTV) and organs at risk (OARs) were calculated for all VMAT plans.

Results: No clinically noticeable differences between the cVMAT and ncVMAT plans were observed in the DV parameters of the PTV. For the lungs, the $V_{13 \text { Gy }}$ and mean dose for ncVMAT plans were smaller than those for cVMAT plans, showing statistically significant differences. For the heart, the values of the maximum dose for cVMAT and ncVMAT plans were $53.8 \pm 2.9$ and $50.9 \pm 3.3 \mathrm{~Gy}$, respectively $(P=0.004)$. For the spinal cord, the values of the maximum dose for CVMAT and ncVMAT plans were $37.1 \pm 5.1$ and $34.7 \pm 5.7 \mathrm{~Gy}$, respectively $(P<0.001)$.

Conclusions: The use of ncVMAT plans provides better PTV coverage and sparing of OARs compared to that of cVMAT plans for long, tube-like esophageal cancer. For esophageal cancer, the ncVMAT plans showed a more favorable plan quality than the cVMAT plans.

Keywords: Dosimetric evaluation, Noncoplanar, Volumetric modulated arc therapy, Esophageal cancer

\section{Introduction}

Esophageal cancer, which mainly occurs in patients aged from 50 to 80 years, is the 11th most common type of cancer in Korea, accounting for approximately $1.7 \%$ of all cancers. An estimated 1,600 new patients are diagnosed with esophageal cancer every year [1]. Treatment options for esophageal cancer are surgery, chemotherapy, or radiotherapy [2-8]. Radiotherapy for inoperable or unresectable esophageal cancer plays a crucial role in achieving optimal tumor control along with reducing normal tissue complications. However, a planning target volume (PTV) is a long, tube-like structure that connects the throat and stomach, as shown in Fig. 1, which creates challenges when planning a treatment. As there are several organs at risk (OARs) in the vicinity of the esophageal cancer, which include the lungs, heart, liver, spinal cord, and stomach, incorrect treatment may cause serious side effects such as radiation pneumonitis and fibrosis in the lungs as well as pericardial effusion, pericarditis, and heart failure [9]. 


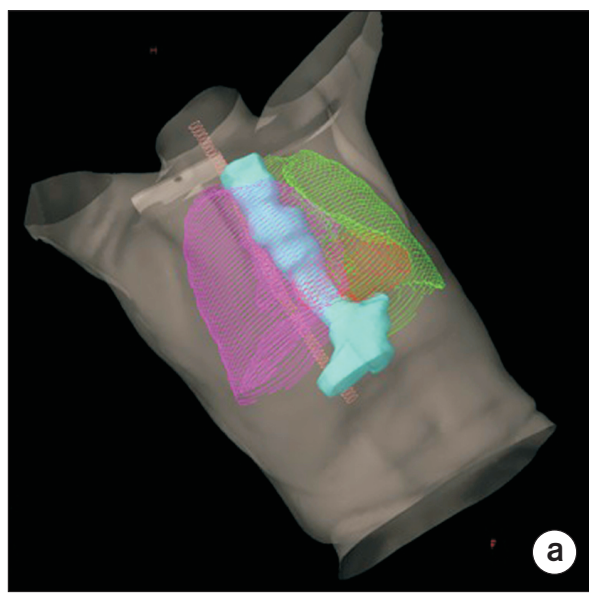

Patient \#1

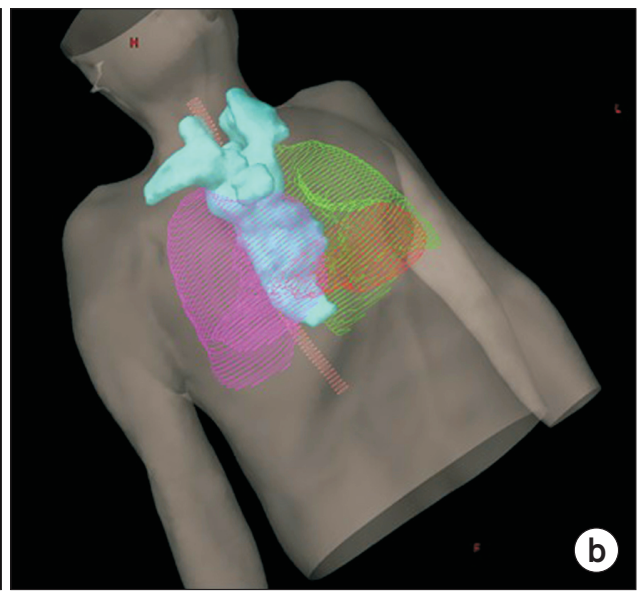

Patient \#3
Fig. 1. Examples of a long, tubeshaped planning target volume (PTV) of esophageal cancer surrounding critical organs at risk such as left and right lungs, heart, and spinal cord for patients \#1 (a) and \#3 (b). Cyan, green, magenta, red, and pink colors represent the PTV, left and right lungs, heart, and spinal cord, respectively.
Several studies have compared various radiotherapy techniques for esophageal cancer, including 3-dimensional conformal radiation therapy (3D CRT), intensity modulated radiation therapy (IMRT), and volumetric modulated arc therapy (VMAT) [2,9-12]. These studies have reported that VMAT plans provide better dose conformity to the PTV while reducing the doses affecting OARs, compared to 3D CRT and IMRT plans [2,9-12]. Furthermore, hybrid IMRT plans that combine static and IMRT beams, and hybrid VMAT plans that combine static beams and VMAT arcs concurrently, have been proposed by several studies to reduce the dose volume applied to normal tissues [9,13-15]. These techniques were found to be superior in dosimetric outcomes when compared to conventional IMRT and VMAT plans.

Li et al. [16] proposed noncoplanar IMRT plans to reduce doses to surrounding OARs while improving PTV coverage for esophageal cancer. They showed that compared to coplanar IMRT plans, noncoplanar IMRT plans reduce the low dose exposure to the lungs, while providing a similar PTV coverage. The disadvantage of noncoplanar IMRT plans is that higher doses are delivered to the heart and spinal cord, making it inadvisable to use noncoplanar IMRT plans for patients with potential cardiovascular and neurological problems. Martini et al. [17] studied noncoplanar VMAT (ncVMAT) plans for patients with esophageal cancer who underwent either definitive or preoperative radiotherapy. Martini et al. [17] evaluated the feasibility, dosimetric results, and clinical outcomes. They showed that, in patients treated with ncVMAT plans using two coplanar and one noncoplanar arc beams, the side effects observed included esophagitis, anorexia, fatigue, nausea, and vomiting. The dosimetric results showed adequate PTV coverage and sparing of normal tissue. They concluded that the use of ncVMAT plans was a feasible, safe, and effective strategy for delivering optimal dose distributions for esophageal cancer patients. Although dosimetric results for ncVMAT plans were investigated in that study, it was a simple feasibility study to confirm the clinical outcome of ncVMAT plans for esophageal cancer, and dosimetric comparisons to other plans were not performed. Therefore, in this study, we compared ncVMAT plans to cVMAT plans by evaluating the dosimetric quality for esophageal cancer with a long, tube-like PTV.

\section{Materials and Methods}

\section{Patient selection and simulation}

Twenty patients with esophageal cancer were retrospectively selected. All patients had been previously treated with radiotherapy at our institution. An approval for this study was obtained from the Institutional Review Board of the Veterans Health Service Medical Center (IRB No. 2020-07011). For selected patients, the mean PTV was $388.3 \pm 163.8$ $\mathrm{cm}^{3}$. The mean PTV craniocaudal extent was $19.4 \pm 3.2 \mathrm{~cm}$, traversing critical normal organs such as lungs, heart, liver, spinal cord, and stomach. All patients underwent comput- 
ed tomography (CT) scans using Brilliance CT Big Bore ${ }^{\mathrm{TM}}$ (Philips, Cleveland, OH, USA) with a slice thickness of 3.0 $\mathrm{mm}$.

\section{Treatment planning}

The prescription dose to the PTV was 60 Gy in 30 fractions for all patients. All VMAT plans were generated with 6-MV photon beams from a Clinac iX with a Millennium 120 multileaf collimator (Varian Medical Systems, Palo Alto, CA, USA). The coplanar VMAT (cVMAT) plans consisted of three coplanar full arc beams (one arc was rotated from $179^{\circ}$ to $181^{\circ}$, whereas the other two arcs were rotated from $181^{\circ}$ to $179^{\circ}$ ). For the cVMAT plans, the collimator angles were set to $3^{\circ}$ or $357^{\circ}$. The ncVMAT plans consisted of two coplanar full arc beams (one arc was rotated from $179^{\circ}$ to $181^{\circ}$, and the other was rotated from $181^{\circ}$ to $179^{\circ}$ ), and one noncoplanar partial arc beam ranging from $45^{\circ}$ to $315^{\circ}$ with a couch rotation angle of $315^{\circ}$. Based on the positional relationship between the PTV and OARs, the original couch angle $\left(315^{\circ}\right)$ was adjusted within $\pm 5^{\circ}$. For the ncVMAT plans, the collimator angles were set to $3^{\circ}$ or $357^{\circ}$ for the two coplanar full arcs and to $45^{\circ} \pm 5^{\circ}$ for the single noncoplanar partial arc. The arc beam geometry for the cVMAT and ncVMAT plans is shown in Fig. 2. Both the cVMAT and ncVMAT plans were optimized with the progressive resolution optimizer 2 (PRO2, ver.8.9; Varian Medical Systems, Palo Alto, CA, USA). The dose distributions were calculated using the anisotropic analytic algorithm (AAA, ver. 8.9; Varian Medical Systems, Palo Alto, CA, USA) with a calculation grid of $1 \mathrm{~mm}$. All plans were normalized so that $100 \%$ of the prescription dose covered $95 \%$ of the PTV.

\section{Dosimetric analysis and evaluation}

The dose-volumetric (DV) parameters were used to assess the dosimetric quality with respect to the PTV coverage and volume of OARs irradiated. For the PTVs, the evaluated DV parameters were the dose received by at least $98 \%$ of the PTV $\mathrm{D}_{98 \%}, \mathrm{D}_{95 \%}, \mathrm{D}_{5 \%}, \mathrm{D}_{2 \%}$, the absolute volume of the PTV irradiated by at least $105 \%$ of the prescription dose $V_{105 \%}$, the minimum dose, the maximum dose, the mean dose, the conformity index $(C I)$, and the homogeneity index $(H I)$. The $C I$ and $H I$ are defined as follows [18-20]:

$$
\begin{aligned}
& \text { Conformity index }(C I)=\frac{\text { Volume of reference isodose }}{\text { Volume of planning target volume }}, \\
& \text { Homogeneity index }(H I)=\frac{D_{5 \%}-D_{98 \%}}{\text { mean dose }}
\end{aligned}
$$

where the volume of reference isodose is the prescription dose.

For both (left and right) lungs, the absolute volume of the normal organ irradiated by up to $30 \mathrm{~Gy}, \mathrm{~V}_{30 \mathrm{~Gy}}$, and the $V_{20 G y}$, the $V_{13 G y}$, the $V_{5 G y}$, the $V_{2.5 G y}$, and the mean dose were all calculated. The DV parameters were also evaluated for whole lungs. For the heart, the dose received by at least 0.1 $\mathrm{cm}^{3}$ volume of the normal organ, $\mathrm{D}_{0.1 \mathrm{~cm}^{3}}, \mathrm{~V}_{40 \mathrm{~Gy}}, \mathrm{~V}_{20 \mathrm{~Gy}}, \mathrm{~V}_{10 \mathrm{~Gy}}$, the minimum dose, the maximum dose, and the mean dose were calculated. For the spinal cord, the $\mathrm{D}_{0.1 \mathrm{~cm}^{3}}$ and the maximum dose were calculated. For the stomach, $\mathrm{V}_{27.2 \mathrm{~Gy}}, \mathrm{~V}_{17.6 \mathrm{~Gy}}$, the minimum dose, the maximum dose, and the mean dose were calculated. For the liver, $\mathrm{V}_{32 \mathrm{~Gy}}, \mathrm{~V}_{28 \mathrm{~Gy}}, \mathrm{~V}_{15 \mathrm{~Gy}}$, the mini-

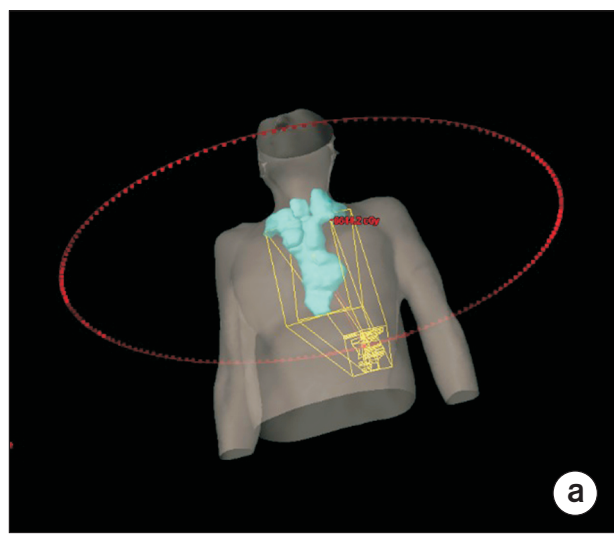

Coplanar VMAT

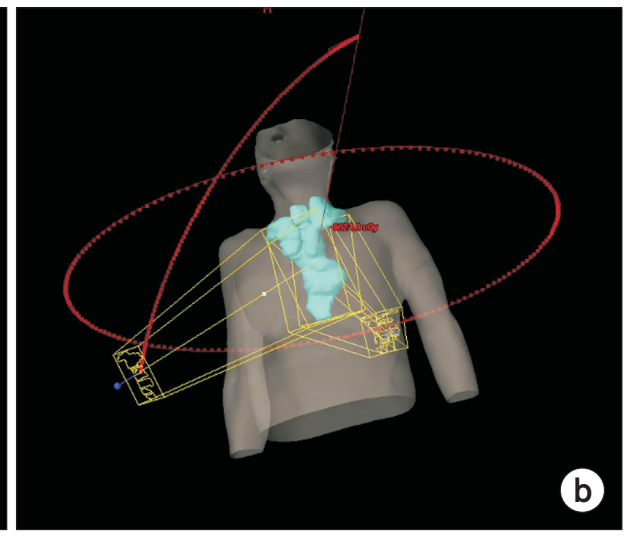

Noncoplanar VMAT
Fig. 2. Arc beam geometry comparison for coplanar (a) and noncoplanar (b) volumetric modulated arc therapy (VMAT) plans. 
Table 1. Average dose-volumetric (DV) parameters of the planning target volume

\begin{tabular}{lccc}
\hline \multicolumn{1}{c}{ DV parameter } & Coplanar VMAT & Noncoplanar VMAT & $P$-value \\
\hline $\mathrm{D}_{98 \%}(\mathrm{~Gy})$ & $58.6 \pm 0.3$ & $58.6 \pm 0.4$ & 0.070 \\
$\mathrm{D}_{95 \%}(\mathrm{~Gy})$ & $60.0 \pm 0.0$ & $60.0 \pm 0.0$ & - \\
$\mathrm{D}_{5 \%}(\mathrm{~Gy})$ & $64.4 \pm 1.0$ & $64.6 \pm 1.0$ & 0.002 \\
$\mathrm{D}_{2 \%}(\mathrm{~Gy})$ & $64.6 \pm 1.1$ & $64.9 \pm 1.0$ & 0.002 \\
$\mathrm{~V}_{105 \%}\left(\mathrm{~cm}^{3}\right)$ & $175.9 \pm 118.5$ & $208.5 \pm 115.0$ & 0.029 \\
Minimum dose (Gy) & $50.7 \pm 3.2$ & $50.8 \pm 2.5$ & 0.737 \\
Maximum dose (Gy) & $66.4 \pm 1.9$ & $66.7 \pm 1.6$ & 0.403 \\
Mean dose (Gy) & $62.8 \pm 0.7$ & $63.0 \pm 0.7$ & 0.005 \\
$\mathrm{CI}$ & $1.026 \pm 0.045$ & $1.028 \pm 0.042$ & 0.330 \\
$\mathrm{HI}$ & $0.069 \pm 0.016$ & $0.071 \pm 0.014$ & 0.102 \\
\hline
\end{tabular}

Values are presented as mean \pm standard deviation.

VMAT, volumetric modulated arc therapy; $\mathrm{D}_{\mathrm{n} \%}$, dose received by at least $\mathrm{n} \%$ volume of the planning target volume; $\mathrm{V}_{\mathrm{n} \%}$, absolute volume of a structure irradiated by at least n\% of the prescription dose; CI, conformity index; HI, homogeneity index.

mum dose, the maximum dose, and the mean dose were calculated.

For the whole body of each patient, $\mathrm{V}_{100 \%}, \mathrm{~V}_{90 \%}, \mathrm{~V}_{70 \%}, \mathrm{~V}_{50 \%}$, $\mathrm{V}_{30 \%}, \mathrm{~V}_{10 \%}$, the mean dose, and the gradient index (GI) were obtained. The GI is defined as follows [21]:

$$
\text { Gradient index }(G I)=\frac{V_{50 \%} \text { of the body }}{V_{100 \%} \text { of the body }}
$$

The mean total monitor units (MUs) were compared for both the cVMAT and ncVMAT plans.

All statistical analyses were performed with paired t-tests to assess whether the observed differences between the cVMAT and ncVMAT plans were statistically significant. A $P$-value of less than 0.05 was considered statistically significant.

\section{Results}

\section{Total MU and DV parameters of the PTV}

The mean total MUs of the cVMAT and the ncVMAT plans were $471.0 \pm 44.2$ and $469.3 \pm 47.6$, respectively $(P=0.850)$.

Table 1 summarizes the DV parameters of the PTV for both the cVMAT and ncVMAT plans. The values of $\mathrm{D}_{98 \%}$ for the two plans were almost identical with no statistical significance. The values of $\mathrm{D}_{95 \%}$ were also identical for both plans. Except for these two DV parameters ( $\mathrm{D}_{98 \%}$ and $\left.\mathrm{D}_{95 \%}\right)$, all other DV parameters for the cVMAT plans were slightly lower than those for the ncVMAT plans. The differences in values of $\mathrm{D}_{5 \%}, \mathrm{D}_{2 \%}, \mathrm{~V}_{105 \%}$, and the mean dose were statistically significant. The PTV conformity and homogeneity for the cVMAT plans were slightly better (lower) than those for the ncVMAT plans; however, the differences were not statistically significant (1.026 vs. 1.028 for CI with $P=0.330$ and 0.069 vs. 0.071 for $\mathrm{HI}$ with $P=0.102$ ). No clinically noticeable differences between the cVMAT and ncVMAT plans were observed in the DV parameters of the PTV.

For dosimetric evaluation, the dose distributions of the cVMAT and ncVMAT plans from representative patient cases (patients 3 and 9) are shown in Fig. 3. The dose-volume histograms for these two patient cases are shown in Fig. 4.

\section{DV parameters of the OARs}

The DV parameters of the OARs for both the cVMAT and the ncVMAT plans are shown in Table 2. The values of $\mathrm{V}_{13 \mathrm{~Gy}}$ and the mean dose values of the left and right lungs for the ncVMAT plans were smaller than those for the cVMAT plans, showing statistically significant differences. The value of $\mathrm{V}_{13 \mathrm{~Gy}}$ of the right lung for the ncVMAT plans was much lower than that for the cVMAT plans $\left(957.7 \mathrm{~cm}^{3}\right.$ for cVMAT plans vs. 900.6 $\mathrm{cm}^{3}$ for ncVMAT plans with $\left.P=0.017\right)$. For both lungs, in general, all DV parameters for the ncVMAT plans were lower than those for the cVMAT plans, except for $\mathrm{V}_{5 \mathrm{~Gy}}$ and $\mathrm{V}_{2.5 \mathrm{~Gy}}$. Among these, the value of $V_{13 \text { Gy }}$ for the ncVMAT plans was much lower than that for the cVMAT plans $\left(1,747.4 \mathrm{~cm}^{3}\right.$ for $\mathrm{cV}$ MAT plans vs. $1,636.4 \mathrm{~cm}^{3}$ for ncVMAT plans with $P=0.002$ ).

For the heart, the values of $\mathrm{V}_{10 \text { Gy }}$ and maximum dose 


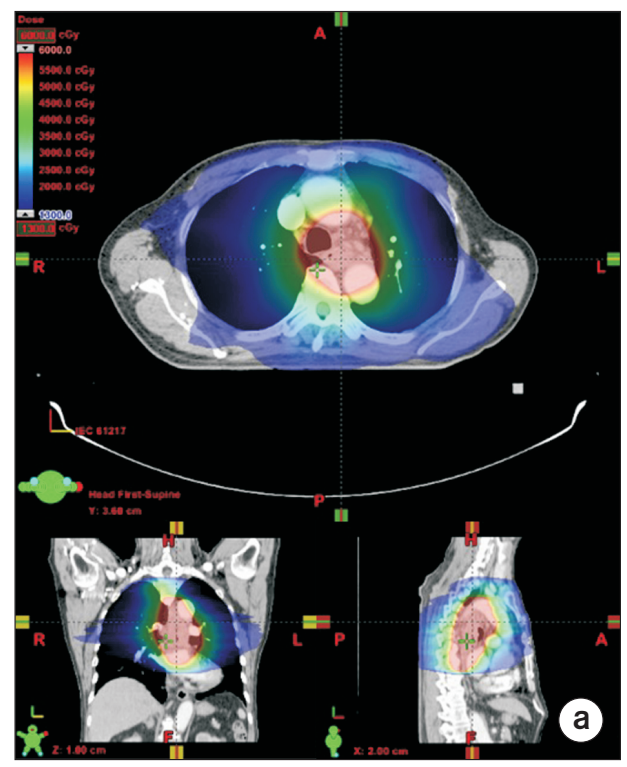

cVMAT (patient \#3)

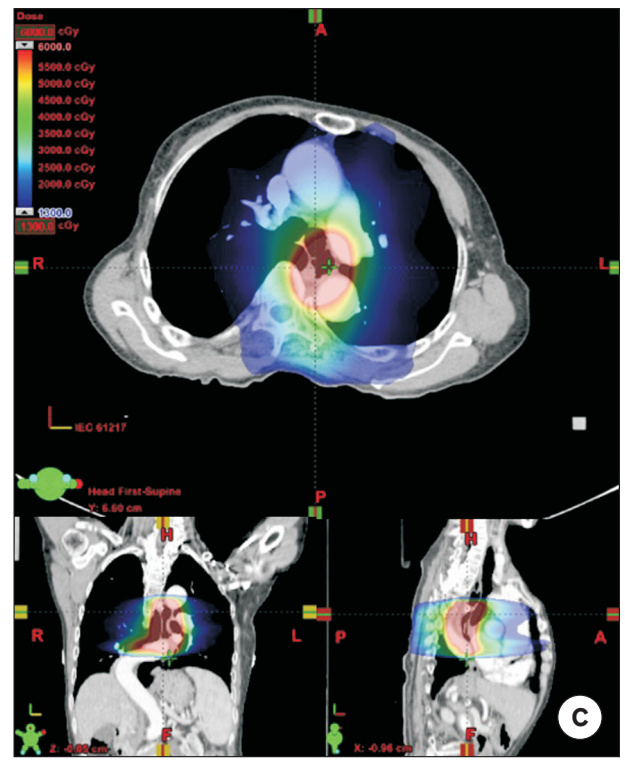

cVMAT (patient \#9)

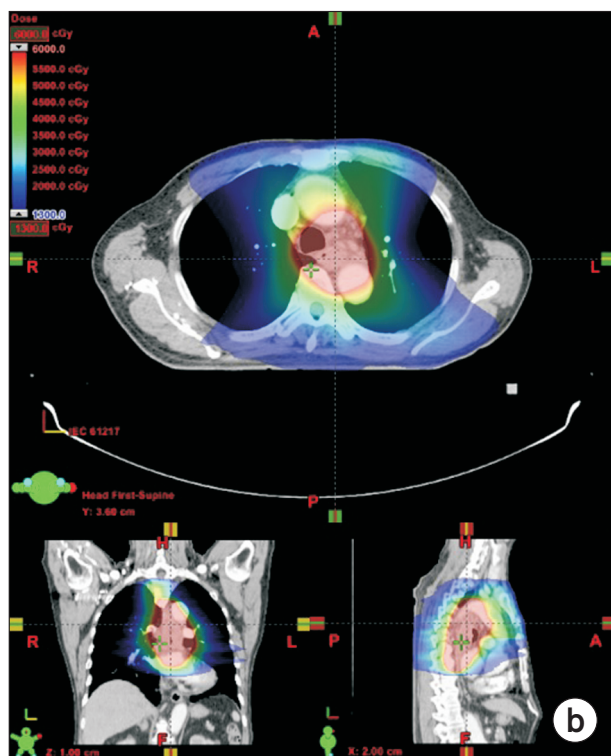

ncVMAT (patient \#3)

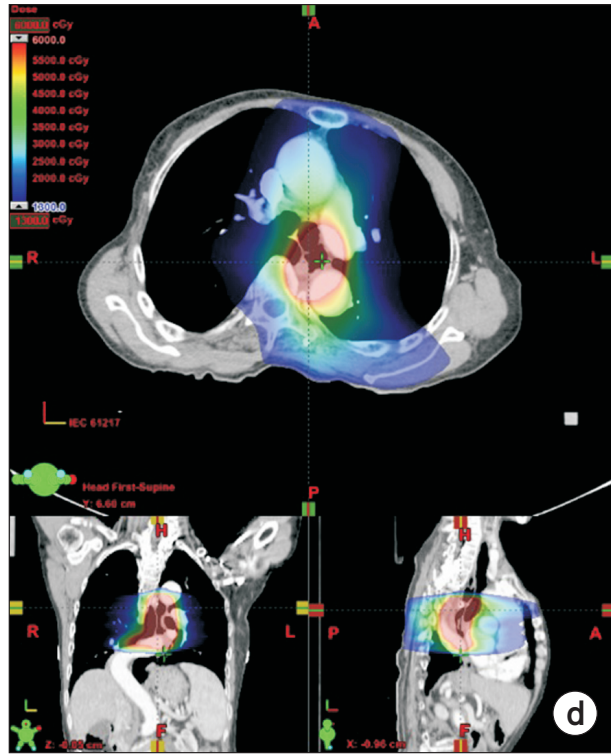

ncVMAT (patient \#9)

Fig. 3. For representative patient cases (patient \#3 and \#9), calculated dose distributions in the axial, coronal, and sagittal views of coplanar volumetric modulated arc therapy (cVMAT) plans (a) and noncoplanar VMAT (ncVMAT) plans (b) of patient \#3, and cVMAT (c) and ncVMAT (d) of patient \#9.

values for the ncVMAT plans were lower than those for the cVMAT plans with the differences being statistically significant ( $P=0.013$ and $P=0.004$, respectively). For the spinal cord, all DV parameters $\left(\mathrm{D}_{0,1 \mathrm{~cm}^{3}}\right.$ and maximum dose) for the ncVMAT plans were much smaller than those for the cVMAT plans, also showing statistically significant differences (all with $P<0.001$ ). The DV parameters for the stomach for the ncVMAT plans were slightly lower than those for the
cVMAT plans; however, none of the differences were statistically significant. For the liver, the values of $\mathrm{V}_{32 \mathrm{~Gy}}$ and $\mathrm{V}_{15 \mathrm{~Gy}}$ for the ncVMAT plans were lower than those for the cVMAT plans with statistically significant differences. The other DV parameters for the liver showed no significant differences between the cVMAT and ncVMAT plans. 


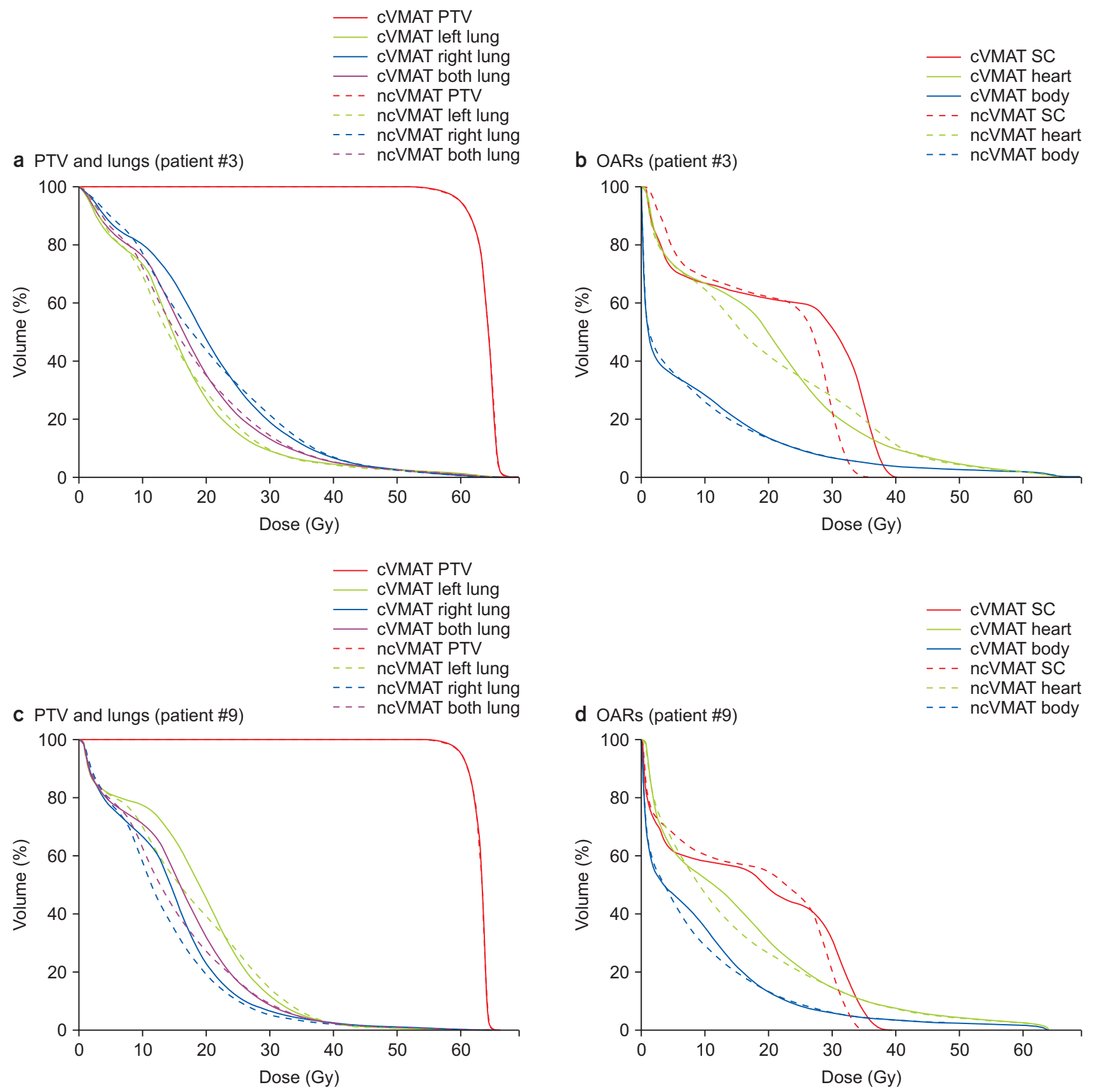

Fig. 4. For both coplanar volumetric modulated arc therapy (cVMAT) and noncoplanar VMAT (ncVMAT) plans, the dose-volume histograms (DVHs) of the planning target volume (PTV) and lungs of the patient \#3 (a) and \#9 (c) are shown. The DVHs of the other organs at risk (OARs) of the patient \#3 (b) and \#9 (d) are shown. The cVMAT and ncVMAT plans are plotted with the solid and dashed lines, respectively. SC, spinal cord.

\section{DV parameters of the whole body}

The DV parameters of the whole body for both the $\mathrm{cV}$ MAT and ncVMAT plans are shown in Table 3. The value of $\mathrm{V}_{100 \%}$ for the ncVMAT plans was slightly higher than that for the cVMAT plans $\left(394.4 \mathrm{~cm}^{3}\right.$ for cVMAT plans vs. $396.1 \mathrm{~cm}^{3}$ for ncVMAT plans with $P=0.015$ ) while the value of $\mathrm{V}_{30 \%}$ for the ncVMAT plans was much lower than that for the $\mathrm{cV}$ MAT plans $\left(3,728.7 \mathrm{~cm}^{3}\right.$ for cVMAT plans vs. $3,640.0 \mathrm{~cm}^{3}$ for ncVMAT plans with $P=0.015)$. The lower dose distribution to the whole body in the ncVMAT plans than in the cVMAT plans is shown in Fig. 3. The differences in the values of 
Table 2. Average dose-volumetric (DV) parameters of normal organs

\begin{tabular}{|c|c|c|c|}
\hline DV parameter & Coplanar VMAT & Noncoplanar VMAT & $P$-value \\
\hline \multicolumn{4}{|l|}{ Left lung } \\
\hline $\mathrm{V}_{30 \text { Gy }}\left(\mathrm{cm}^{3}\right)$ & $111.3 \pm 63.1$ & $112.9 \pm 72.2$ & 0.688 \\
\hline $\mathrm{V}_{20 \mathrm{~Gy}}\left(\mathrm{~cm}^{3}\right)$ & $414.6 \pm 205.3$ & $396.2 \pm 202.1$ & 0.159 \\
\hline $\mathrm{V}_{13 \mathrm{~Gy}}\left(\mathrm{~cm}^{3}\right)$ & $790.6 \pm 293.2$ & $736.6 \pm 291.4$ & 0.001 \\
\hline $\mathrm{V}_{5 \mathrm{~Gy}}\left(\mathrm{~cm}^{3}\right)$ & $1,080.0 \pm 390.8$ & $1,082.6 \pm 385.5$ & 0.501 \\
\hline $\mathrm{V}_{2.5 \mathrm{~Gy}}\left(\mathrm{~cm}^{3}\right)$ & $1,160.6 \pm 409.8$ & $1,167.0 \pm 405.2$ & 0.113 \\
\hline Mean dose (Gy) & $16.2 \pm 4.1$ & $15.8 \pm 4.2$ & 0.017 \\
\hline \multicolumn{4}{|l|}{ Right lung } \\
\hline $\mathrm{V}_{30 \mathrm{~Gy}}\left(\mathrm{~cm}^{3}\right)$ & $136.0 \pm 86.2$ & $133.1 \pm 86.2$ & 0.353 \\
\hline $\mathrm{V}_{20 \mathrm{~Gy}}\left(\mathrm{~cm}^{3}\right)$ & $434.4 \pm 237.5$ & $417.2 \pm 219.5$ & 0.189 \\
\hline $\mathrm{V}_{13 \mathrm{~Gy}}\left(\mathrm{~cm}^{3}\right)$ & $957.7 \pm 364.9$ & $900.6 \pm 361.8$ & 0.017 \\
\hline $\mathrm{V}_{5 \mathrm{~Gy}}\left(\mathrm{~cm}^{3}\right)$ & $1,396.5 \pm 416.6$ & $1,406.5 \pm 425.8$ & 0.095 \\
\hline $\mathrm{V}_{2.5 \mathrm{~Gy}}\left(\mathrm{~cm}^{3}\right)$ & $1,505.2 \pm 409.8$ & $1,518.4 \pm 416.5$ & 0.088 \\
\hline Mean dose (Gy) & $15.5 \pm 3.6$ & $15.2 \pm 3.5$ & 0.026 \\
\hline \multicolumn{4}{|l|}{ Both lungs } \\
\hline $\mathrm{V}_{30 \mathrm{~Gy}}\left(\mathrm{~cm}^{3}\right)$ & $246.7 \pm 134.1$ & $245.3 \pm 139.7$ & 0.785 \\
\hline $\mathrm{V}_{20 \mathrm{~Gy}}\left(\mathrm{~cm}^{3}\right)$ & $848.2 \pm 424.7$ & $812.7 \pm 391.5$ & 0.113 \\
\hline $\mathrm{V}_{13 \mathrm{~Gy}}\left(\mathrm{~cm}^{3}\right)$ & $1,747.4 \pm 636.0$ & $1,636.4 \pm 631.6$ & 0.002 \\
\hline $\mathrm{V}_{5 \mathrm{~Gy}}\left(\mathrm{~cm}^{3}\right)$ & $2,475.5 \pm 752.9$ & $2,488.2 \pm 754.7$ & 0.008 \\
\hline $\mathrm{V}_{2.5 \mathrm{~Gy}}\left(\mathrm{~cm}^{3}\right)$ & $2,664.9 \pm 754.5$ & $2,684.4 \pm 752.6$ & 0.008 \\
\hline Mean dose (Gy) & $15.7 \pm 3.6$ & $15.3 \pm 3.6$ & 0.006 \\
\hline \multicolumn{4}{|l|}{ Heart } \\
\hline $\mathrm{D}_{0.1 \mathrm{~cm}^{3}}(\mathrm{~Gy})$ & $50.3 \pm 4.8$ & $50.4 \pm 5.0$ & 0.633 \\
\hline $\mathrm{V}_{40 \mathrm{~Gy}}\left(\mathrm{~cm}^{3}\right)$ & $50.6 \pm 38.1$ & $51.5 \pm 40.1$ & 0.602 \\
\hline $\mathrm{V}_{20 \mathrm{~Gy}}\left(\mathrm{~cm}^{3}\right)$ & $283.1 \pm 171.9$ & $271.5 \pm 160.1$ & 0.257 \\
\hline $\mathrm{V}_{10 \mathrm{~Gy}}\left(\mathrm{~cm}^{3}\right)$ & $519.2 \pm 252.2$ & $501.4 \pm 253.6$ & 0.013 \\
\hline Minimum dose (Gy) & $3.2 \pm 3.7$ & $2.8 \pm 3.2$ & 0.275 \\
\hline Maximum dose (Gy) & $53.8 \pm 2.9$ & $50.9 \pm 3.3$ & 0.004 \\
\hline Mean dose (Gy) & $18.0 \pm 6.6$ & $17.8 \pm 6.6$ & 0.473 \\
\hline \multicolumn{4}{|l|}{ Spinal cord } \\
\hline $\mathrm{D}_{0.1 \mathrm{~cm}^{3}}(\mathrm{~Gy})$ & $35.9 \pm 4.9$ & $33.6 \pm 5.4$ & $<0.001$ \\
\hline Maximum dose (Gy) & $37.1 \pm 5.1$ & $34.7 \pm 5.7$ & $<0.001$ \\
\hline \multicolumn{4}{|l|}{ Stomach } \\
\hline $\mathrm{V}_{27.2 \mathrm{~Gy}}\left(\mathrm{~cm}^{3}\right)$ & $67.7 \pm 28.5$ & $66.8 \pm 34.1$ & 0.852 \\
\hline $\mathrm{V}_{17.6 \mathrm{~Gy}}\left(\mathrm{~cm}^{3}\right)$ & $96.4 \pm 39.8$ & $90.3 \pm 44.6$ & 0.184 \\
\hline Minimum dose (Gy) & $8.1 \pm 10.5$ & $8.0 \pm 9.9$ & 0.843 \\
\hline Maximum dose (Gy) & $61.3 \pm 5.6$ & $61.4 \pm 5.5$ & 0.699 \\
\hline Mean dose (Gy) & $27.0 \pm 15.0$ & $26.8 \pm 15.3$ & 0.687 \\
\hline \multicolumn{4}{|l|}{ Liver } \\
\hline $\mathrm{V}_{32 \mathrm{~Gy}}\left(\mathrm{~cm}^{3}\right)$ & $68.7 \pm 52.7$ & $57.8 \pm 53.6$ & 0.027 \\
\hline $\mathrm{V}_{28 \mathrm{~Gy}}\left(\mathrm{~cm}^{3}\right)$ & $100.6 \pm 73.1$ & $100.8 \pm 71.5$ & 0.080 \\
\hline $\mathrm{V}_{15 \text { Gy }}\left(\mathrm{cm}^{3}\right)$ & $399.6 \pm 267.6$ & $348.7 \pm 245.5$ & 0.015 \\
\hline Minimum dose (Gy) & $0.4 \pm 0.3$ & $0.4 \pm 0.3$ & 0.694 \\
\hline Maximum dose (Gy) & $57.3 \pm 17.2$ & $58.2 \pm 15.5$ & 0.193 \\
\hline Mean dose (Gy) & $12.5 \pm 6.6$ & $12.2 \pm 6.2$ & 0.226 \\
\hline
\end{tabular}

VMAT, volumetric modulated arc therapy; $\mathrm{V}_{\mathrm{n} \text { Gy }}$, absolute volume of a structure irradiated by at least $\mathrm{n}$ Gy; $\mathrm{D}_{\mathrm{n} \mathrm{cm}^{3}}$, dose received by at least $\mathrm{n}$ $\mathrm{cm}^{3}$ volume of a structure. 
Table 3. Average dose-volumetric (DV) parameters of the body

\begin{tabular}{lccc}
\hline \multicolumn{1}{c}{ DV parameter } & Coplanar VMAT & Noncoplanar VMAT & $P$-value \\
\hline $\mathrm{V}_{100 \%}\left(\mathrm{~cm}^{3}\right)$ & $394.4 \pm 161.1$ & $396.1 \pm 163.2$ & 0.015 \\
$\mathrm{~V}_{90 \%}\left(\mathrm{~cm}^{3}\right)$ & $507.2 \pm 26.2$ & $509.8 \pm 211.0$ & 0.137 \\
$\mathrm{~V}_{70 \%}\left(\mathrm{~cm}^{3}\right)$ & $765.5 \pm 309.6$ & $780.6 \pm 327.0$ & 0.118 \\
$\mathrm{~V}_{50 \%}\left(\mathrm{~cm}^{3}\right)$ & $1,431.0 \pm 578.1$ & $1,458.7 \pm 609.9$ & 0.422 \\
$\mathrm{~V}_{30 \%}\left(\mathrm{~cm}^{3}\right)$ & $3,728.7 \pm 1,540.8$ & $3,640.0 \pm 1,530.4$ & 0.001 \\
$\mathrm{~V}_{10 \%}\left(\mathrm{~cm}^{3}\right)$ & $9,803.8 \pm 3,382.6$ & $9,754.9 \pm 3,431.2$ & 0.320 \\
Mean dose (Gy) & $8.8 \pm 2.3$ & $8.7 \pm 2.3$ & 0.004 \\
GI & $3.7 \pm 0.3$ & $3.7 \pm 0.2$ & 0.257 \\
\hline
\end{tabular}

VMAT, volumetric modulated arc therapy; $\mathrm{V}_{\mathrm{n} \%}$, absolute volume of a structure irradiated by at least $\mathrm{n} \%$ of the prescription dose; GI, gradient index.

$\mathrm{V}_{90 \%}, \mathrm{~V}_{70 \%}, \mathrm{~V}_{50 \%}$, and $\mathrm{V}_{10 \%}$ between the cVMAT and the $\mathrm{ncV}$ MAT plans had no statistical significance. The mean dose to the whole body for ncVMAT plans was slightly lower than that for cVMAT plans, showing a statistically significant difference $(P=0.004)$. The values of $G I$, which indicate the dose fall-off outside the PTV, were similar for both plans.

\section{Discussion}

In this study, we compared the potential dosimetric improvements of the ncVMAT plans with those of the cVMAT plans, for the long, tube-like PTV of esophageal cancer. The cVMAT plans with three coplanar full arc beams and the ncVMAT plans with two coplanar full arc beams and one noncoplanar partial beam were used for planning. For the noncoplanar beam, the couch rotation angles were adjusted to $315^{\circ} \pm 5^{\circ}$ to avoid collision between the patient on the couch and the gantry. For dosimetric evaluation, the DV parameters of the PTV and OARs and total MUs for each VMAT plan were obtained.

To date, this is the first study that attempted to evaluate the dosimetric superiority of ncVMAT plans in patients with long, tube-like esophageal targets for a large group of patients $(n=20)$. In a similar study, Li et al. [16] investigated the dose distributions around esophageal cancer and the surrounding OARs using coplanar IMRT and noncoplanar IMRT. They reported that the conformity and mean dose of the PTV were comparable between the coplanar IMRT and noncoplanar IMRT plans while the values of $\mathrm{V}_{5 \%}, \mathrm{~V}_{10 \%}, \mathrm{~V}_{20 \%}$, and the mean dose for lungs using the noncoplanar IMRT plans decreased by $16.3,10.6,5.8$, and $248.7 \mathrm{~cm}^{3}$, respec- tively, compared to those for the coplanar IMRT plans. The results of the present study are consistent with those found in earlier studies. The ncVMAT plans reduce the doses to OARs such as the lungs, heart, and spinal cord, as shown in this study. We found that the maximum doses to both the spinal cord and heart were dramatically reduced in the ncVMAT plans. For representative patient cases (patients 4 and 7) in this study, when using the ncVMAT plans, the maximum dose differences of 6.4 and 3.0 Gy were observed in volumes in the spinal cord and heart, respectively. We also found that low doses to the lungs and liver were significantly reduced for the ncVMAT plans. However, the values of $\mathrm{V}_{105 \%}$ of the PTV and $\mathrm{V}_{100 \%}$ of the whole body were increased for the ncVMAT plans. Nevertheless, these findings show some potential benefit of using the ncVMAT plans.

Except for the spinal cord and heart, there were minor improvements in dose distribution from the ncVMAT plans over the cVMAT plans in this study. This is mainly because the noncoplanar arc beam used in this study was limited. When using large noncoplanar angles, the probability of collision between the patient and the gantry increased. Theoretically, the $4 \pi$ technique could provide significant OAR sparing compared to both the cVMAT and ncVMAT plans and then allow substantial dose escalation to the PTV $[22,23]$. Woods et al. [22] demonstrated that the $4 \pi$ technique reduced $50 \%$ of the prescription doses by $\sim 23 \%$, compared to both cVMAT and ncVMAT plans. However, this technique significantly increases both the treatment time and the probability of collision between the patient and the gantry. In the clinic, we need to determine the noncoplanar arc beam configuration for effective treatment that repre- 
sents a compromise to account for the combination of the gantry rotation angle and the couch angle.

Noncoplanar techniques have been widely examined for spherical targets for brain tumor radiotherapy, lung stereotactic ablative radiotherapy (SABR), and liver SABR patients, showing dosimetric benefits to either the PTV or the OARs [9,22,24-27]. However, there are few dosimetric studies for large, irregularly shaped PTVs such as head, neck, and esophageal cancer. Gayen et al. [28] compared the dosimetric plan quality of the ncVMAT and cVMAT plans for head and neck malignancy and then demonstrated that ncVMAT plans provide better PTV coverage and OAR sparing, compared to cVMAT plans. Nevertheless, treatment of large, irregularly shaped head, neck, and esophageal cancer with noncoplanar beams is challenging due to the complicated positional relationship between the PTVs and the surrounding OARs. Further studies to prove the superiority of ncVMAT plans over cVMAT plans according to the size and shape of the PTV will be performed in the future.

\section{Conclusions}

The use of ncVMAT plans with two coplanar full arc beams and one noncoplanar partial beam provides better PTV coverage and OARs sparing, compared to that of cVMAT plans with three full arc beams for long, tube-like esophageal cancer. Furthermore, this technique can be adopted in the clinic for large, irregularly shaped targets with caution.

\section{Acknowledgements}

This study was supported by Basic Science Research Program through the National Research Foundation of Korea (NRF) funded by the Ministry of Education (NRF-2017R1D1A1B03036093) and by a VHS Medical Center Research Grant, Republic of Korea (grant number: VHSMC 20056).

\section{Conflicts of Interest}

The authors have nothing to disclose.

\section{Availability of Data and Materials}

All relevant data are within the paper and its Supporting Information files.

\section{Ethics Approval and Consent to Participate}

The study was approved by the Institutional Review Board of the Veterans Health Service Medical Center (IRB No. 2020-07-011).

\section{References}

1. Statistics Korea. Statistics Korea 2020 [cited 2020 Jan 16]. Available from: http://www.index.go.kr/.

2. Fawaz ZS, Kazandjian S, Tsui JM, Devic DS, LecavalierBarsoum M, Vuong T, et al. What is the optimal radiation technique for esophageal cancer? A dosimetric comparison of four techniques. Cureus. 2018;10:e2985.

3. Zhang M, Wu AJ. Radiation techniques for esophageal cancer. Chin Clin Oncol. 2017;6:45.

4. Park J, Yea JW. Whole brain radiotherapy using four-field box technique with tilting baseplate for parotid gland sparing. Radiat Oncol J. 2019;37:22-29.

5. Ahn YC. Less is more: role of additional chemotherapy to concurrent chemoradiotherapy in locoregionally advanced nasopharyngeal cancer management. Radiat Oncol J. 2019; $37: 67-72$

6. Huh SJ, Park W, Choi DH. Recent trends in intensity-modulated radiation therapy use in Korea. Radiat Oncol J. 2019; 37:249-253.

7. Jeong C, Park JW, Kwak J, Song SY, Cho B. Volumetric-modulated arc radiotherapy using knowledge-based planning: application to spine stereotactic body radiotherapy. Prog Med Phys. 2019;30:94-103.

8. Rahman MM, Kim CH, Kim S. Mid-term performance of clinical LINAC in volumetric modulated arc therapy. J Radiat Prot Res. 2019;44:43-52.

9. Mayo CS, Urie MM, Fitzgerald TJ, Ding L, Lo YC, Bogdanov M. Hybrid IMRT for treatment of cancers of the lung and esophagus. Int J Radiat Oncol Biol Phys. 2008;71:1408-1418.

10. Zhang WZ, Zhai TT, Lu JY, Chen JZ, Chen ZJ, Li DR, et al. Volumetric modulated arc therapy vs. c-IMRT for the treat- 
ment of upper thoracic esophageal cancer. PLoS One. 2015; 10:e0121385.

11. Yin L, Wu H, Gong J, Geng JH, Jiang F, Shi AH, et al. Volumetric-modulated arc therapy vs. c-IMRT in esophageal cancer: a treatment planning comparison. World J Gastroenterol. 2012;18:5266-5275.

12. Xu C, Xi M, Komaki R, Balter PA, Huang M, Hobbs BP, et al. Dosimetric and clinical outcomes after volumetric modulated arc therapy for carcinoma of the thoracic esophagus. Adv Radiat Oncol. 2017;2:325-332.

13. Chan OS, Lee MC, Hung AW, Chang AT, Yeung RM, Lee AW. The superiority of hybrid-volumetric arc therapy (VMAT) technique over double arcs VMAT and 3D-conformal technique in the treatment of locally advanced nonsmall cell lung cancer--a planning study. Radiother Oncol. 2011;101:298-302.

14. Verbakel WF, van Reij E, Ladenius-Lischer I, Cuijpers JP, Slotman BJ, Senan S. Clinical application of a novel hybrid intensity-modulated radiotherapy technique for stage III lung cancer and dosimetric comparison with four other techniques. Int J Radiat Oncol Biol Phys. 2012;83:e297-e303.

15. Abbas AS, Moseley D, Kassam Z, Kim SM, Cho C. Volumetric-modulated arc therapy for the treatment of a large planning target volume in thoracic esophageal cancer. J Appl Clin Med Phys. 2013;14:4269.

16. Li Y, Liu B, Zhai F, Yang Y, Liu M, Bao C, et al. Dosimetric study of coplanar and non-coplanar intensity-modulated radiation therapy planning for esophageal carcinoma. Int J Med Phys Clin Eng Radiat Oncol. 2013;2:133-138.

17. Martini S, Arcadipane F, Strignano P, Spadi R, Contu V, Fiandra C, et al. Volumetric modulated arc therapy (VMAT) in the treatment of esophageal cancer patients. Med Oncol. 2018;35:150.

18. Shaw E, Kline R, Gillin M, Souhami L, Hirschfeld A, Dinapoli R, et al. Radiation Therapy Oncology Group: radiosurgery quality assurance guidelines. Int J Radiat Oncol Biol Phys. 1993;27:1231-1239.

19. Zhang P, Happersett L, Hunt M, Jackson A, Zelefsky M,
Mageras G. Volumetric modulated arc therapy: planning and evaluation for prostate cancer cases. Int J Radiat Oncol Biol Phys. 2010;76:1456-1462.

20. Kim ST, An HJ, Kim JI, Yoo JR, Kim HJ, Park JM. Non-coplanar VMAT plans for lung SABR to reduce dose to the heart: a planning study. Br J Radiol. 2020;93:20190596.

21. Paddick I, Lippitz B. A simple dose gradient measurement tool to complement the conformity index. J Neurosurg. 2006;105 Suppl:194-201.

22. Woods K, Nguyen D, Tran A, Yu VY, Cao M, Niu T, et al. Viability of non-coplanar VMAT for liver SBRT as compared to coplanar VMAT and beam orientation optimized $4 \pi$ IMRT. Adv Radiat Oncol. 2016;1:67-75.

23. Wild E, Bangert M, Nill S, Oelfke U. Noncoplanar VMAT for nasopharyngeal tumors: plan quality versus treatment time. Med Phys. 2015;42:2157-2168.

24. Dong P, Lee P, Ruan D, Long T, Romeijn E, Low DA, et al. $4 \pi$ noncoplanar stereotactic body radiation therapy for centrally located or larger lung tumors. Int J Radiat Oncol Biol Phys. 2013;86:407-413.

25. Zhang S, Yang R, Shi C, Li J, Zhuang H, Tian S, et al. Noncoplanar VMAT for brain metastases: a plan quality and delivery efficiency comparison with coplanar VMAT, IMRT, and CyberKnife. Technol Cancer Res Treat. 2019;18: 1533033819871621.

26. Smyth G, Evans PM, Bamber JC, Mandeville HC, Welsh LC, Saran FH, et al. Non-coplanar trajectories to improve organ at risk sparing in volumetric modulated arc therapy for primary brain tumors. Radiother Oncol. 2016;121:124-131.

27. Sharfo AW, Dirkx ML, Breedveld S, Méndez Romero A, Heijmen BJ. VMAT plus a few computer-optimized non-coplanar IMRT beams (VMAT+) tested for liver SBRT. Radiother Oncol. 2017;123:49-56.

28. Gayen S, Kombathula SH, Manna S, Varshney S, Pareek P. Dosimetric comparison of coplanar and non-coplanar volumetric-modulated arc therapy in head and neck cancer treated with radiotherapy. Radiat Oncol J. 2020;38:138147. 